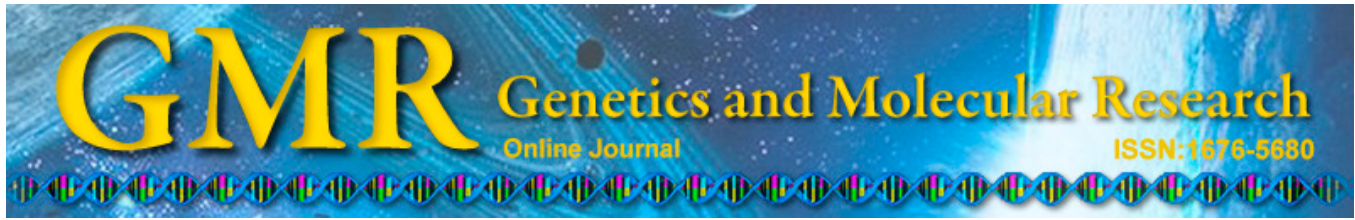

\title{
Characterization and development of chloroplast microsatellite markers for Gossypium hirsutum, and cross-species amplification in other Gossypium species
}

\author{
X.Y. Cai, F. Liu, Z.L. Zhou, X.X. Wang, C.Y. Wang, Y.H. Wang and \\ K.B. Wang
}

State Key Laboratory of Cotton Biology, Institute of Cotton Research of the Chinese Academy of Agricultural Sciences, Anyang, China

Corresponding author: K.B. Wang

E-mail: wkbcri@163.com

Genet. Mol. Res. 14 (4): 11924-11932 (2015)

Received February 7, 2015

Accepted May 29, 2015

Published October 5, 2015

DOI http://dx.doi.org/10.4238/2015.October.5.6

\begin{abstract}
Cotton is an important economic crop worldwide; its fiber, commonly known as cotton lint, is the main natural source for the textile industry. Sixty chloroplast microsatellites were identified and characterized from the complete sequence of the Gossypium hirsutum chloroplast genome using a bioinformatic approach. Twenty chloroplast microsatellite loci were polymorphic in the 66 Gossypium germplasm accessions. A total of 85 alleles were detected, with allele numbers varying from 2-7 per locus. Polymorphism information content varied from $0.02-0.66$, with a mean of 0.48 . Additionally, transferability of the 20 polymorphic chloroplast microsatellite primers was evaluated in other 31 Gossypium species. Sixteen markers were successfully amplified across all species tested, while the remaining 4 markers cross-amplified in most species tested. These polymorphic chloroplast microsatellite markers may be useful tool for studies of individual
\end{abstract}


identification, genetic diversity, evolution, conservation genetics, and molecular breeding in Gossypium.

Key words: Chloroplast microsatellite; Gossypium; Polymorphism; Transferability

\section{INTRODUCTION}

Cotton is one of the most important economic crops worldwide. Its fiber is the main natural source for the textile industry. The genus Gossypium consists of over 45 diploid $(2 \mathrm{~N}=2 \mathrm{x}=26)$ and 5 tetraploid $(2 \mathrm{~N}=4 \mathrm{x}=52)$ species distributed widely throughout the tropical and subtropical regions of the world (Wang et al., 2012). Diploid species have been classified into 8 genome groups designated $\mathrm{A}-\mathrm{G}$ and $\mathrm{K}$, while the 5 tetraploid species were designated as the $\mathrm{AD}$ genome group based on morphologic traits, cytogenetic pairing, and fertility of interspecific hybrids (Fryxell, 1992; Wendel and Albert, 1992). Four species are cultivated, including Gossypium hirsutum, Gossypium barbadense, Gossypium herbaceum, and Gossypium arboretum, while the others are wild species. Upland cotton (G. hirsutum) accounts for more than $90 \%$ of cotton fiber output, whereas sea cotton (G. barbadense) produces long-staple fibers and accounts for approximately $8 \%$ of cotton production. Wild cotton species have many valuable agronomic traits and abundant gene resources (Wang, 2007). However, many wild Gossypium species are at risk of extinction because of habitat deterioration, human activities, and low germination rates under natural conditions because of its hard episperm. Extensive field studies showed that wild G. tomentosum accessions, endemic to Hawaii, have sharply reduced in number.

Chloroplast microsatellites (cpSSRs) were developed by Powell et al. (1995) and successfully applied in population genetic studies of pines. Compared to the nuclear genome, the chloroplast genome is typically non-recombinant, uniparentally inherited, has a high copy number, and shows relatively conserved gene order, making it possible to detect relatively rapidly mutating sites in the chloroplast genome using cpSSR markers. To date, cpSSR markers have been developed for a variety of species and were shown to be invaluable in studying the evolution, differentiation, genetic diversity, and genetic structure of plant populations (Powell et al., 1995; Ishii and McCouch, 2000; Xu et al., 2002; Deguilloux et al., 2003; Dzialuk et al., 2009; Xue et al., 2012; Wang, 2013; Sugiura et al., 2014).

Numerous studies have examined the ecological and evolution aspects of Gossypium based on allozymes (Wendel et al., 1994), restriction fragment length polymorphism (Wendel and Brubaker, 1993), random-amplified polymorphic DNA (Tatineni et al., 1996; Lu and Myers, 2002), amplified fragment length polymorphism (Pillay and Myers, 1999; Rana et al., 2005), and simple sequence repeats (SSRs) (Wu et al., 2007; Yu et al., 2012). The results of these studies were complementary and largely congruent with existing genome designations and geographical distributions. However, there are still some ambiguities in the systematic relationships of these species and which genomes were more ancestral among extant cotton species. Additional markers should be developed to study the genetic diversity and phylogeny of Gossypium. In this study, we developed and characterized a set of polymorphic cpSSR markers from the complete chloroplast genome of G. hirsutum and tested their transferability to other Gossypium species, which will be useful in addressing questions related to the genetic diversity and phylogeny of Gossypium, thus making them important for evolutionary and conservation purposes. 


\section{MATERIAL AND METHODS}

Sixty-six germplasm accessions of Gossypium belonging to 32 species were used to evaluate the polymorphism and transferability of the cpSSR markers (Table 1). All voucher samples were maintained in the National Wild Cotton Nursery (Sanya, Hainan Province, China), and supervised by the Institute of Cotton Research of the Chinese Academy of Agricultural Sciences.

DNA was extracted from fresh leaves using the CTAB method as described by Zhang and Stewart (2000), with some modifications. DNA concentration and quality were evaluated by $1.0 \%(\mathrm{w} / \mathrm{v})$ agarose gel electrophoresis and by spectrophotometry at $260 / 280 \mathrm{~nm}$. The DNA solutions were stored at $-20^{\circ} \mathrm{C}$.

The complete sequence of the G. hirsutum chloroplast genome (accession No. NC_007944) was downloaded from GenBank (www.ncbi.nlm.nih.gov). Mononucleotide cpSSRs were located using the program Tandem Repeats Finder 4.04 (Benson, 1999), and polynucleotide cpSSRs were searched in SSRhunter (Li and Wan, 2005). The labile length of the motif was considered to be 8 or more nucleotides (Rose and Falush, 1998; Raubeson et al., 2007). In this study, 10 nucleotides was the threshold for motifs used to search for mononucleotide repeats. Motifs of 5, 4, and 3 were used to search for dinucleotide, trinucleotide, and tetranucleotide repeats, respectively. In addition, analysis of the structure of chloroplast genome was performed using the DOGMA software (http//:dogma.ccbb.utexas.edu/).

Primers were designed from the sequences of cpSSRs using the Primer Premier 5.0 software (Premier Biosoft, Palo Alto, CA, USA), based on G+C content, Tm, and the lack of secondary structure. Primers were designed in the 18-22-bp range to yield amplification products of $100-400$ bases.

DNA from 64 individuals including 32 species was amplified by polymerase chain reaction in a $10-\mu \mathrm{L}$ reaction volume containing $1 \mu \mathrm{L} 10 \mathrm{X}$ PCR Buffer, $1.75 \mathrm{mM} \mathrm{MgCl}, 175$ $\mu \mathrm{M}$ dNTPs, 0.5 U TransStart Taq DNA polymerase (TransGen, Biotech, Beijing, China), and approximately $30 \mathrm{ng}$ DNA template. The amplification was performed in a PT600 Programmable Thermal Controller (TaKaRa, Shiga, Japan) following a touchdown cycling program: $5 \mathrm{~min}$ denaturation at $95^{\circ} \mathrm{C}$, followed by 10 cycles of $30 \mathrm{~s}$ denaturation at $95^{\circ} \mathrm{C}$, decreasing temperatures from $60^{\circ}$ to $50^{\circ} \mathrm{C}$ by $1^{\circ} \mathrm{C}$ every cycle for $30 \mathrm{~s}, 50 \mathrm{~s}$ elongation at $72^{\circ} \mathrm{C}$, followed by 30 cycles of denaturation at $95^{\circ} \mathrm{C}, 30 \mathrm{~s}$ annealing at $50^{\circ} \mathrm{C}$, and $50 \mathrm{~s}$ elongation at $72^{\circ} \mathrm{C}$, and a final extension for $5 \mathrm{~min}$ at $72^{\circ} \mathrm{C}$. The amplification products were separated on $8 \%$ denatured polyacrylamide gel. Gels were visualized by silver-staining. The molecular sizes of the amplicons were estimated by comparison with a 500-bp DNA ladder (Invitrogen, Carlsbad, CA, USA).

\section{RESULTS}

A total of $60 \mathrm{cpSSRs}$ were developed from the whole chloroplast genome sequence of G. hirsutum (Table 2). We found 1 long single-copy sequence, 1 short single-copy sequence, and 2 inverted repeat in the G. hirsutum chloroplast genome. cpSSRs were mainly distributed randomly across the single-copy region with both the long single-copy sequence (49) and short single-copy sequence (9) of the G. hirsutum chloroplast genome, but only 2 cpSSRs in the inverted repeat region. There were 43 mononucleotide cpSSRs (mainly A/T), 15 dinucleotide cpSSRs, and 2 trinucleotide cpSSRs, with lengths of at least 10 nucleotides. Although cpSSRs were present throughout the whole chloroplast genome, they were not evenly distributed, appearing to be concentrated in several areas. 
Table 1. Sixty-six cotton germplasm accessions used in this study.

\begin{tabular}{|c|c|c|c|}
\hline Sample No. & Material & Genome & Geographic origin \\
\hline 1 & G. hirsutum L. 'Pengpengmian' & $\mathrm{AD}_{1}$ & Hainan, China \\
\hline 2 & G. hirsutum L. 'zhong12' & $\mathrm{AD}^{1}$ & China \\
\hline 3 & G. hirsutum L. 'zhong16' & $\mathrm{AD}^{1}$ & China \\
\hline 4 & G. hirsutum L. 'TM-1' & $\mathrm{AD}^{1}$ & America \\
\hline 5 & G. hirsutum L. 'T582' & $\mathrm{AD}^{1}$ & America \\
\hline 6 & G. barbadense L. 'H7124' & $\mathrm{AD}^{1}$ & South America \\
\hline 7 & G. barbadense L. 'Yuanmo' & $\mathrm{AD}^{2}$ & Yunnan, China \\
\hline 8 & G. barbadense L. 'Mile' & $\mathrm{AD}_{2}^{2}$ & Yunnan, China \\
\hline 9 & G. barbadense L. 'Kaiyuanlihe' & $\mathrm{AD}_{2}^{2}$ & Yunnan, China \\
\hline 10 & G. barbadense L. 'cheli' & $\mathrm{AD}_{2}^{2}$ & Yunnan, China \\
\hline 11 & G. barbadense L. 'Kaiyuanlianhe' & $\mathrm{AD}_{2}^{2}$ & Yunnan, China \\
\hline 12 & G. barbadense L. 'Arablianhe' & $\mathrm{AD}_{2}^{2}$ & Yunnan, China \\
\hline 13 & G. tomentosum Nuttall ex Seemann & $\mathrm{AD}_{3}^{2}$ & Hawaii Islands \\
\hline 14 & G. tomentosum Nuttall ex Seemann & $\mathrm{AD}_{3}^{3}$ & Hawaii Islands \\
\hline 15 & G. mustelinum Miers ex Watt & $\mathrm{AD}_{4}$ & Brazil \\
\hline 16 & G. mustelinum Miers ex Watt & $\mathrm{AD}^{4}$ & Brazil \\
\hline 17 & G. darwinii Watt & $\mathrm{AD}_{5}^{4}$ & Galapagos Islands \\
\hline 18 & G. darwinii Watt & $\mathrm{AD}_{5}^{5}$ & Galapagos Islands \\
\hline 19 & G. darwinii Watt & $\mathrm{AD}_{5}^{5}$ & Galapagos Islands \\
\hline 20 & G. herbaceum L. 'jinta' & $\mathrm{A}_{1}$ & Asia \\
\hline 21 & G. herbaceum L. 'Africanum' & $\mathrm{A}_{1}$ & Africa \\
\hline 22 & G. arboreum L. 'Rozi' & $\mathrm{A}_{2}$ & Asia \\
\hline 23 & G. arboreum L. 'zhongya1' & $\mathrm{A}_{2}^{2}$ & Asia and Africa \\
\hline 24 & G.arboreum L. 'AK235' & $\mathrm{A}_{2}^{2}$ & Asia \\
\hline 25 & G. anomalum Wawra \& Peyritsch & $\mathrm{B}^{2}$ & Africa \\
\hline 26 & G. anomalum Wawra \& Peyritsch & $\mathrm{B}_{1}^{1}$ & Africa \\
\hline 27 & G. capitis-viridis Mauer & $\mathrm{B}_{2}^{1}$ & Cape Verde Islands \\
\hline 28 & G. capitis-viridis Mauer & $\mathrm{B}_{2}^{2}$ & Cape Verde Islands \\
\hline 29 & G. sturtianum Willis & $\mathrm{C}_{1}^{2}$ & Central Australia \\
\hline 30 & G. sturtianum Willis & $\mathrm{C}_{1}^{1}$ & Central Australia \\
\hline 31 & G. nandewarense Derera & $\mathrm{C}_{1}^{1}$ & Southeast Australia \\
\hline 32 & G. robinsonii Mueller & $\mathrm{C}_{2}^{1-\mathrm{n}}$ & West Australia \\
\hline 33 & G. thurberi Todaro & $\mathrm{D}_{1}^{2}$ & Arizona \\
\hline 34 & G. armourianum Kearney & $D_{2+1}^{1}$ & Mexica and California \\
\hline 35 & G. harknessii Brandegee & $\mathrm{D}_{2-2}^{2-1}$ & Mexica and California \\
\hline 36 & G. davidsonii Kellogg & $\mathrm{D}_{3-\mathrm{d}}^{2-2}$ & Mexica and California \\
\hline 37 & G. davidsonii Kellogg & $\mathrm{D}_{3}^{3-\mathrm{d}}$ & Mexica and California \\
\hline 38 & G. davidsonii Kellogg & $\mathrm{D}_{3}^{3-0}$ & Mexica and California \\
\hline 39 & G. klotzschianum Andersson & $D_{3-k}^{3-d}$ & Galapagos Islands \\
\hline 40 & G. aridum (Rose \& Standley) Skovsted & $\mathrm{D}_{4}^{3-k}$ & Mexica and California \\
\hline 41 & G. raimondii Ulbrich & $\mathrm{D}_{5}^{4}$ & Peru \\
\hline 42 & G. raimondii Ulbrich & $\mathrm{D}_{5}^{5}$ & Peru \\
\hline 43 & G. raimondii Ulbrich & $\mathrm{D}_{5}^{5}$ & Peru \\
\hline 44 & G. gossypioides (Ulbrich) Standley & $\mathrm{D}^{3}$ & Mexica \\
\hline 45 & G. gossypioides (Ulbrich) Standley & $\mathrm{D}^{6}$ & Mexica \\
\hline 46 & G. gossypioides (Ulbrich) Standley & $\mathrm{D}^{6}$ & Mexica \\
\hline 47 & G. lobatum Gentry & $\mathrm{D}_{7}^{6}$ & Michoacan \\
\hline 48 & G. trilobum (DC.) Skovsted & $\mathrm{D}_{8}^{7}$ & West Mexica \\
\hline 49 & G. laxum Phillips & $\mathrm{D}_{9}^{8}$ & Guerrero \\
\hline 50 & G. schwendimanii Fryxell & $\mathrm{D}_{1}$ & Mexica \\
\hline 51 & G. stocksii Masters in Hooker & $\mathrm{E}_{1}^{11}$ & Arabia \\
\hline 52 & G. stocksii Masters in Hooker & $\mathrm{E}_{1}^{1}$ & Arabia \\
\hline 53 & G. stocksii Masters in Hooker & $E_{1}^{1}$ & Arabia \\
\hline 54 & G. somalense (Gurke) Hutchinson & $\mathrm{E}_{2}$ & North Africa \\
\hline 55 & G. somalense (Gurke) Hutchinson & $\mathrm{E}_{2}^{2}$ & North Africa \\
\hline 56 & G. areysianum Deflers & $\mathrm{E}_{3}^{2}$ & South Yemen \\
\hline 57 & G. incanum (Schwartz) Hillcoat & $\mathrm{E}_{4}^{3}$ & South Africa \\
\hline 58 & G. incanum (Schwartz) Hillcoat & $\mathrm{E}_{4}^{4}$ & South Africa \\
\hline 59 & G. longicalyx Hutchinson \& Lee & $\mathrm{F}_{1}^{4}$ & East Africa \\
\hline 60 & G. bickii Prokhanov & $\mathrm{G}_{1}^{1}$ & Central Australia \\
\hline 61 & G. bickii Prokhanov & $\mathrm{G}_{\mathrm{T}}$ & Central Australia \\
\hline 62 & G. bickii Prokhanov & $\mathrm{G}_{2}$ & Central Australia \\
\hline 63 & G. australe Mueller & $\mathrm{G}_{2}^{2}$ & Australia \\
\hline 64 & G. nelsonii Fryxell & $\mathrm{G}_{3}^{2}$ & Central Australia \\
\hline 65 & G. nelsonii Fryxell & $\mathrm{G}_{3}^{3}$ & Central Australia \\
\hline 66 & G. nelsonii Fryxell & $\mathrm{G}_{3}^{3}$ & Central Australia \\
\hline
\end{tabular}


X.Y. Cai et al.

Table 2. Distribution of $60 \mathrm{cpSSRs}$ in the Gossypium hirsutum chloroplast genome.

\begin{tabular}{|c|c|c|c|c|}
\hline No. & Motif & SSR start (bp) & SSR end (bp) & Distribution area \\
\hline 1 & $\mathrm{~A}_{10}$ & 1906 & 1916 & LSC \\
\hline 2 & $\mathrm{~T}_{11}^{10}$ & 2419 & 2430 & LSC \\
\hline 3 & $\mathrm{C}_{10}$ & 5505 & 5515 & LSC \\
\hline 4 & $\left(\mathrm{~T}_{8}\right) \mathrm{CG}\left(\mathrm{A}_{10}\right)$ & 5664 & 5684 & LSC \\
\hline 5 & $\mathrm{~A}_{10}$ & 6657 & 6667 & LSC \\
\hline 6 & $\mathrm{~A}_{10}^{10}$ & 7620 & 7630 & LSC \\
\hline 7 & $\mathrm{~A}_{10}$ & 8897 & 8907 & LSC \\
\hline 8 & $\mathrm{~T}_{10}^{10}$ & 9429 & 9439 & LSC \\
\hline 9 & $\left(\mathrm{C}_{10}\right)\left(\mathrm{T}_{7}\right)^{10} \ldots\left(\mathrm{T}_{9}\right)$ & 14715 & 14749 & LSC \\
\hline 10 & $\mathrm{~A}_{12}$ & 14924 & 14936 & LSC \\
\hline 11 & $\mathrm{~T}_{11}^{12}$ & 19341 & 19352 & LSC \\
\hline 12 & $\mathrm{~T}_{11}^{11}$ & 21770 & 21781 & LSC \\
\hline 13 & $\mathrm{~T}_{12}^{11}$ & 27055 & 27067 & LSC \\
\hline 14 & $A_{10} \ldots G_{8}$ & 38033 & 38057 & LSC \\
\hline 15 & $\mathrm{~A}_{12}$ & 52682 & 52694 & LSC \\
\hline 16 & $\mathrm{~T}_{11}^{12}$ & 54773 & 54784 & LSC \\
\hline 17 & $\mathrm{~T}_{12}^{11}$ & 66007 & 66019 & LSC \\
\hline 18 & $\left(\mathrm{~T}_{10}\right) \mathrm{C}\left(\mathrm{T}_{6}\right)$ & 67266 & 67283 & LSC \\
\hline 19 & $\mathrm{G}_{13}$ & 67304 & 67317 & LSC \\
\hline 20 & $\mathrm{~T}_{10}^{13}$ & 69432 & 69442 & LSC \\
\hline 21 & $\mathrm{~T}_{12}^{10}$ & 70335 & 70347 & LSC \\
\hline 22 & $\left(\mathrm{~T}_{14}\right) \mathrm{AG}\left(\mathrm{T}_{0}\right)$ & 71286 & 71311 & LSC \\
\hline 23 & $\mathrm{~T}_{10}$ & 71629 & 71639 & LSC \\
\hline 24 & $\mathrm{~T}_{10}^{10}$ & 74663 & 74673 & LSC \\
\hline 25 & $\mathrm{~A}_{10} \ldots \mathrm{A}_{8}$ & 74965 & 74988 & LSC \\
\hline 26 & $\mathrm{~A}_{10}$ & 79410 & 79420 & LSC \\
\hline 27 & $\mathrm{G}_{10}$ & 80014 & 80024 & LSC \\
\hline 28 & $\mathrm{~A}_{10}$ & 80784 & 80794 & LSC \\
\hline 29 & $\mathrm{~T}_{10}^{10}$ & 81140 & 81150 & LSC \\
\hline 30 & $\mathrm{~A}_{10}^{10}$ & 82150 & 82160 & LSC \\
\hline 31 & $\left(\mathrm{~T}_{6}\right) \mathrm{C}\left(\mathrm{T}_{10}\right)$ & 84443 & 84460 & LSC \\
\hline 32 & $\mathrm{~T}_{10}$ & 84959 & 84969 & LSC \\
\hline 33 & $A_{11}^{10}$ & 85490 & 85501 & LSC \\
\hline 34 & $\mathrm{~T}_{12}^{11}$ & 103834 & 103846 & IR \\
\hline 35 & $\mathrm{~A}_{12}$ & 115241 & 115253 & SSC \\
\hline 36 & $\mathrm{~A}_{10}$ & 115362 & 115373 & SSC \\
\hline 37 & $\mathrm{~A}_{11}$ & 115885 & 115896 & SSC \\
\hline 38 & $\left(\mathrm{~A}_{11}\right) \mathrm{T}\left(\mathrm{A}_{7}\right)$ & 117135 & 117154 & SSC \\
\hline 39 & $\mathrm{~A}_{10}$ & 118295 & 118305 & SSC \\
\hline 40 & $\mathrm{~A}_{11}^{10}$ & 120246 & 120257 & SSC \\
\hline 41 & $\mathrm{~T}_{10}^{11}$ & 125787 & 125797 & SSC \\
\hline 42 & $\mathrm{~A}_{10}^{10}$ & 131300 & 131310 & SSC \\
\hline 43 & $\mathrm{~A}_{12}$ & 145199 & 145211 & IR \\
\hline 44 & $(\mathrm{CT})_{7} \mathrm{~T}_{8}$ & 17110 & 17132 & LSC \\
\hline 45 & $(\mathrm{AT})_{5}$ & 20722 & 20732 & LSC \\
\hline 46 & $(\mathrm{AT})_{5}$ & 28125 & 28134 & LSC \\
\hline 47 & $(\mathrm{TG})_{5} \mathrm{~T}_{9}$ & 32721 & 32740 & LSC \\
\hline 48 & $(\mathrm{TA})_{6}$ & 33024 & 33035 & LSC \\
\hline 49 & $(\mathrm{AT})_{5}$ & 44519 & 44528 & LSC \\
\hline 50 & $(\mathrm{AT})_{5}$ & 46839 & 46848 & LSC \\
\hline 51 & $(\mathrm{AT})_{5}$ & 48436 & 48445 & LSC \\
\hline 52 & $(\mathrm{TA})_{5}$ & 49137 & 49146 & LSC \\
\hline 53 & $(\mathrm{AT})_{5}$ & 51060 & 51069 & LSC \\
\hline 54 & $(\mathrm{TA})_{5}$ & 58355 & 58364 & LSC \\
\hline 55 & $(\mathrm{AT})_{5}$ & 64971 & 64980 & LSC \\
\hline 56 & $(\mathrm{TC})_{5}$ & 65263 & 65272 & LSC \\
\hline 57 & $(\mathrm{AT})_{5}$ & 86188 & 86197 & LSC \\
\hline 58 & $(\mathrm{TA})_{5}^{5}$ & 131174 & 131183 & SSC \\
\hline 59 & $(\mathrm{AAT})_{4}$ & 13592 & 13603 & LSC \\
\hline 60 & $(\mathrm{ATA})_{4}$ & 48444 & 48455 & LSC \\
\hline
\end{tabular}

$\mathrm{LSC}=$ long single-copy sequence in the chloroplast genome; $\mathrm{SSC}=$ short single-copy sequence in the chloroplast genome; IR $=2$ inverted repeats in the chloroplast genome. 
Of the $60 \mathrm{cpSSRs}, 40$ were amenable to primer design. Primers could not be designed for the remaining sequences because of the unavailability of a sufficient number of bases to meet the primer designing criteria or because of biased base compositions in the flanking nucleotide sequences. A set of 40 primer pairs were synthesized and checked for $5 \mathrm{G}$. hirsutum accessions (Sample No. 1-5). Of the $40 \mathrm{cpSSR}$ primers, 20 primers producing repeatable banding patterns were polymorphic (Table 3 ). Other primers that showed no amplification, no multiple bands, or no pronounced stutters were excluded from further study.

\begin{tabular}{|c|c|c|c|c|c|c|}
\hline Markers & Motif & Primer sequences $\left(5^{\prime}-3^{\prime}\right)$ & $\mathrm{Ta}\left({ }^{\circ} \mathrm{C}\right)$ & Size (bp) & $N_{\mathrm{A}}$ & PIC \\
\hline ср01 & $\mathrm{A}_{10}$ & $\begin{array}{l}\text { F: AGTCGGATGGAGTAGATAA } \\
\text { R: TCAGGCAGTAAGAATAAGAG }\end{array}$ & TD & 207 & 5 & 0.61 \\
\hline сp02 & $\mathrm{T}_{11}$ & $\begin{array}{l}\text { F: TGTTCTTCTTCCGTTTCC } \\
\text { R: TTATTGACCGATTTGTGC }\end{array}$ & TD & 211 & 2 & 0.02 \\
\hline сp03 & $\left(\mathrm{T}_{8}\right) \mathrm{CG}\left(\mathrm{A}_{10}\right)$ & $\begin{array}{l}\text { F: ATCCATAAACCAGCAAATC } \\
\text { R: TTCTAAGCGAGACAAACA }\end{array}$ & TD & 184 & 5 & 0.62 \\
\hline сp04 & $\mathrm{A}_{10}$ & $\begin{array}{l}\text { F: GTTTGGCAAGCTGCTGTA } \\
\text { R: GACTCTAATCTAAATAGGGTTCT }\end{array}$ & TD & 233 & 2 & 0.06 \\
\hline сp05 & $\mathrm{T}_{11}$ & $\begin{array}{l}\text { F: AAGCGACCAGTTCCAATC } \\
\text { R: ATCTAGGCCAGAAGCAGA }\end{array}$ & TD & 498 & 2 & 0.25 \\
\hline ср06 & $\mathrm{T}_{12}$ & $\begin{array}{l}\text { F: CTTTACTCTTTCTACCCATCA } \\
\text { R: AATACGCCCAGTTGCTTC }\end{array}$ & TD & 326 & 3 & 0.51 \\
\hline ср07 & $\left(\mathrm{T}_{10}\right) \mathrm{C}\left(\mathrm{T}_{6}\right)$ & $\begin{array}{l}\text { F: TGTTCTTCTTGGCATCTA } \\
\text { R: ATACTCCCTGTTTGTTCC }\end{array}$ & TD & 481 & 6 & 0.63 \\
\hline сp08 & $\mathrm{T}_{10}$ & $\begin{array}{l}\text { F: GGCCTAGAAACGGAAGA } \\
\text { R: AATAGAGCGGCAGGACAA }\end{array}$ & TD & 396 & 4 & 0.56 \\
\hline ср09 & $\mathrm{T}_{12}$ & $\begin{array}{l}\text { F: CAATACAGACGTGGTGAT } \\
\text { R: GAGCAGTAACGAGGGAGT }\end{array}$ & TD & 439 & 5 & 0.62 \\
\hline сp10 & $\left(\mathrm{T}_{14}\right) \mathrm{AG}\left(\mathrm{T}_{9}\right)$ & $\begin{array}{l}\text { F: GGTTCTTTAGCGGGTTTA } \\
\text { R: GAGTTTCGGTGAGGTTGA }\end{array}$ & TD & 254 & 7 & 0.58 \\
\hline cp11 & $\mathrm{T}_{10}$ & $\begin{array}{l}\text { F: GAGAAATGTGGCTGGAAC } \\
\text { R: CTGGAAATACCCGTTGAT }\end{array}$ & TD & 380 & 6 & 0.66 \\
\hline сp12 & $\mathrm{T}_{10}$ & $\begin{array}{l}\text { F: CACATAATGAGGCGAGAA } \\
\text { R: GCTGAATCACAGACGAAA }\end{array}$ & TD & 285 & 5 & 0.61 \\
\hline сp13 & $\mathrm{A}_{10}$ & $\begin{array}{l}\text { F: AAGCAAGGCATTTCTGGT } \\
\text { R: TCTTACGGAGCCCTATTT }\end{array}$ & TD & 433 & 5 & 0.58 \\
\hline cp14 & $\mathrm{T}_{10}$ & $\begin{array}{l}\text { F: ATAGGCTGGTTCGCTTGA } \\
\text { R: TGCTTTATGGGTCGTCTT }\end{array}$ & TD & 335 & 4 & 0.56 \\
\hline cp15 & $\mathrm{T}_{10}$ & $\begin{array}{l}\text { F: ACCGTAGGGCAGGAGGAGT } \\
\text { R: AATGACAGACCGGGAGGC }\end{array}$ & TD & 162 & 2 & 0.11 \\
\hline сp16 & $\mathrm{T}_{10}$ & $\begin{array}{l}\text { F: GCATTTCGTATAGAGGTT } \\
\text { R: TAAGACGAGACCCAAGTA }\end{array}$ & TD & 203 & 3 & 0.45 \\
\hline сp17 & $\mathrm{A}_{11}$ & $\begin{array}{l}\text { F: CAATGTAACAAAGGACGAA } \\
\text { R: TTATGAAATGAGCGGAGT }\end{array}$ & TD & 214 & 3 & 0.40 \\
\hline cp18 & $\mathrm{T}_{12}$ & $\begin{array}{l}\text { F: CCGAAGAGTAACTAGGACA } \\
\text { R: GAATGATGGAAGGGAATA }\end{array}$ & TD & 333 & 4 & 0.54 \\
\hline сp19 & $\mathrm{A}_{13}$ & $\begin{array}{l}\text { F: ACCGTCACCCATTCTAAC } \\
\text { R: ACTTTCTTGATTCCCATC }\end{array}$ & TD & 322 & 5 & 0.67 \\
\hline ср20 & $\mathrm{A}_{10}$ & $\begin{array}{l}\text { F: GGGAAAATCGCATAAAAT } \\
\text { R: AAAACTGGGCGTAGATAG }\end{array}$ & TD & 253 & 6 & 0.65 \\
\hline
\end{tabular}

$\mathrm{Ta}=$ annealing temperature; $\mathrm{TD}=$ touchdown $\mathrm{PCR}$ program with a temperature from $60^{\circ} \mathrm{C}$, decreasing by $1^{\circ} \mathrm{C}$ per cycle until $50^{\circ} \mathrm{C} ; N_{\mathrm{A}}=$ number of alleles; $\mathrm{PIC}=$ polymorphism information content.

Polymorphism of the $20 \mathrm{cpSSR}$ primer pairs was tested across all samples. A total of 85 alleles were detected in 66 Gossypium germplasm accessions. The number of alleles per locus ranged from 2-7, with an average of 4.20 per locus. Polymorphism information content (PIC) varied from $0.02-0.66$, with a mean of 0.48 (Table 3 ). 
In this study, the transferability of the 20 polymorphic cpSSR primers was tested in other 31 Gossypium species. Sixteen cpSSR primer pairs successfully amplified across all species tested, while the remaining 4 primer pairs (cp07, cp11, cp12, and cp20) produced amplification products across most species (Table 4). The results showed that all cpSSR markers had good transferability in the Gossypium species. All of these polymorphic cpSSR markers from G. hirsutum were transferable to other Gossypium species, suggesting that these cpSSR markers could be applied to evaluating the genetic diversity of Gossypium.

\begin{tabular}{|c|c|c|c|c|c|c|c|c|c|c|c|c|c|c|c|c|c|c|c|c|}
\hline Species & ср01 & ср02 & ср03 & ср04 & ср05 & ср06 & ср07 & ср08 & ср09 & cp10 & cp11 & cp12 & cp13 & cp14 & cp15 & cp16 & cp17 & cp18 & cp19 & cp20 \\
\hline G. hirsutum & + & + & + & + & + & + & + & + & + & + & + & + & + & + & + & + & + & + & + & + \\
\hline G. barbadense & + & + & + & + & + & + & + & + & + & + & + & + & + & + & + & + & + & + & + & + \\
\hline G. tomentosum & + & + & + & + & + & + & + & + & + & + & + & + & + & + & + & + & + & + & + & + \\
\hline G. mustelinum & + & + & + & + & + & + & + & + & + & + & + & + & + & + & + & + & + & + & + & + \\
\hline G. darwinii & + & + & + & + & + & + & + & + & + & + & + & + & + & + & + & + & + & + & + & + \\
\hline G. herbaceum & + & + & + & + & + & + & + & + & + & + & + & + & + & + & + & + & + & + & + & + \\
\hline G. arboreum & + & + & + & + & + & + & + & + & + & + & + & + & + & + & + & + & + & + & + & + \\
\hline G. anomalum & + & + & + & + & + & + & + & + & + & + & + & + & + & + & + & + & + & + & + & + \\
\hline G. capitis-viridis & + & + & + & + & + & + & + & + & + & + & + & + & + & + & + & + & + & + & + & + \\
\hline G. sturtianum & + & + & + & + & + & + & + & + & + & + & + & + & + & + & + & + & + & + & + & + \\
\hline G. nandewarense & + & + & + & + & + & + & + & + & + & + & + & + & + & + & + & + & + & + & + & + \\
\hline G. robinsonii & + & + & + & + & + & + & + & + & + & + & + & + & + & + & + & + & + & + & + & + \\
\hline G. thurberi & + & + & + & + & + & + & + & + & + & + & + & + & + & + & + & + & + & + & + & + \\
\hline G. armourianum & + & + & + & + & + & + & + & + & + & + & + & + & + & + & + & + & + & + & + & + \\
\hline G. harknessii & + & + & + & + & + & + & + & + & + & + & + & + & + & + & + & + & + & + & + & + \\
\hline G. davidsonii & + & + & + & + & + & + & + & + & + & + & + & + & + & + & + & + & + & + & + & + \\
\hline G. klotzschianum & + & + & + & + & + & + & + & + & + & + & + & + & + & + & + & + & + & + & + & + \\
\hline G. aridum & + & + & + & + & + & + & + & + & + & + & - & - & + & + & + & + & + & + & + & + \\
\hline G. raimondii & + & + & + & + & + & + & + & + & + & + & + & + & + & + & + & + & + & + & + & + \\
\hline G. gossypioides & + & + & + & + & + & + & + & + & + & + & + & + & + & + & + & + & + & + & + & - \\
\hline G. lobatum & + & + & + & + & + & + & + & + & + & + & + & - & + & + & + & + & + & + & + & + \\
\hline G. trilobum & + & + & + & + & + & + & + & + & + & + & + & + & + & + & + & + & + & + & + & + \\
\hline G. laxum & + & + & + & + & + & + & + & + & + & + & + & + & + & + & + & + & + & + & + & + \\
\hline G. schwendimanii & $i+$ & + & + & + & + & + & + & + & + & + & + & + & + & + & + & + & + & + & + & + \\
\hline G. stocksii & + & + & + & + & + & + & + & + & + & + & + & + & + & + & + & + & + & + & + & - \\
\hline G. somalense & + & + & + & + & + & + & - & + & + & + & + & - & + & + & + & + & + & + & + & - \\
\hline G. areysianum & + & + & + & + & + & + & + & + & + & + & + & + & + & + & + & + & + & + & + & + \\
\hline G. incanum & + & + & + & + & + & + & + & + & + & + & + & + & + & + & + & + & + & + & + & + \\
\hline G. longicalyx & + & + & + & + & + & + & + & + & + & + & + & + & + & + & + & + & + & + & + & + \\
\hline G. bickii & + & + & + & + & + & + & - & + & + & + & + & + & + & + & + & + & + & + & + & + \\
\hline G. australe & + & + & + & + & + & + & + & + & + & + & + & + & + & + & + & + & + & + & + & + \\
\hline G. nelsonii & + & + & + & + & + & + & + & + & + & + & + & + & + & + & + & + & + & + & + & - \\
\hline
\end{tabular}

(+) PCR amplification; (-) no PCR amplification.

\section{DISCUSSION}

In the present study, we found $60 \mathrm{cpSSRs}$ in the G. hirsutum chloroplast genome and analyzed the characteristics of their distribution. We identified cpSSRs throughout the whole chloroplast genome, but they were not evenly distributed, appearing to be concentrated in several areas. This result was consistent with those found in other plants (Wang et al., 2006; Pian et al., 2008; Hu et al., 2009; Pan et al., 2014). In addition, there were clear differences in the cpSSR number in different plants. The plant chloroplast genome size is generally 120-160 $\mathrm{kb}$. The chloroplast genome sizes in A. thaliana, C. sativus, M. truncatula, $V$. unguiculata, and G. hirsutum are 154,155,124, 152, and $160 \mathrm{~kb}$, and they contain 84, 227, 241, 52, and 
60 cpSSRs, respectively. Therefore, no significant positive correlation was found between chloroplast genome size and cpSSR number.

The PIC value of a marker provides an estimation of the discrimination power in accessions by considering the number of alleles and the relative frequency of each allele (Smith et al., 2000). This helps in choosing markers for evaluating germplasms. In this study, 14 (70\% of 20$)$ cpSSR markers were highly informative (PIC $>0.5), 3(15 \%$ of 20$) \mathrm{cpSSR}$ markers were moderately informative $(0.25<\mathrm{PIC}<0.5)$, and $3(15 \%$ of 20$) \mathrm{cpSSR}$ markers were lowly informative (PIC $<0.25$ ) (Vigouroux et al., 2002). The number of alleles per locus in this study ranged from 2-7 (mean $=4.25)$, with a mean PIC value of 0.48 . Compared with the alleles and PIC values in other plants described in previous studies, such as those by Yang et al., 2008 (alleles =2-6); Ginwal et al., 2011 (alleles = 2-3); Wang, 2013 (alleles = 1-3), this higher number of alleles and PIC value were not unexpected, as the Gossypium accessions were natural populations, and the genetic bottleneck caused by human-mediated selection pressure to accumulate a particular type of alleles governing various traits has not yet been applied (Iqbal et al., 2001; Vigouroux et al., 2002).

Because the chloroplast genome is highly conservative, cpSSR primers show good transferability between different species (Diekmann et al., 2012; Xue et al., 2012). In this study, transferability of cpSSR primers was tested using 32 cotton species. Twenty polymorphic cpSSR markers developed from $G$. hirsutum were transferable to all or most of the 32 Gossypium species because the flanking regions of cpSSR loci are conserved. These results suggest that all novel cpSSR markers can be used to analyze genetic diversity and population structure in Gossypium.

\section{ACKNOWLEDGMENTS}

Research supported by the National High Technology Research and Development of China (\#2013AA102601) and Projects in the National Science and Technology Pillar Program (\#2013BAD01B03).

\section{REFERENCES}

Benson G (1999). Tandem repeats finder: a program to analyze DNA sequences. Nucleic Acids Res. 27: 573-580.

Deguilloux MF, Dumolin-Lapègue S, Gielly L, Grivet D, et al. (2003). A set of primers for the amplification of chloroplast microsatellites in Quercus. Mol. Ecol. Notes 3: 24-27.

Diekmann K, Hodkinson TR and Barth S (2012). New chloroplast microsatellite markers suitable for assessing genetic diversity of Lolium perenne and other related grass species. Ann. Bot. 110: 1327-1339.

Dzialuk A, Muchewicz E, Boratyński A, Montserrat JM, et al. (2009). Genetic variation of Pinus uncinata (Pinaceae) in the Pyrenees determined with cpSSR markers. Plant Syst. Evol. 277: 197-205.

Fryxell PA (1992). A revised taxonomic interpretation of Gossypium L. (Malvaceae). Rheedea 2: 108-165.

Ginwal HS, Mittal N, Tomar A and Varshney VK (2011). Population genetic structure and diversity of high value vulnerable medicinal plant Acorus calamus in India using RAPD and chloroplast microsatellite markers. J. Forest. Res. 22: 367-377.

Hu JB, Zhou XY and Li JW (2009). Development of novel chloroplast microsatellite markers for Cucumis from sequence database. Biol. Plantarum 53: 793-796.

Iqbal MJ, Reddy OUK, El-Zik KM and Pepper AE (2001). A genetic bottleneck in the 'evolution under domestication' of Upland cotton Gossypium hirsutum L. examined using DNA fingerprinting. Theor. Appl. Genet. 103: 547-554.

Ishii T and McCouch SR (2000). Microsatellites and microsynteny in the chloroplast genomes of Oryza and eight other Gramineae species. Theor. Appl. Genet. 100: 1257-1266.

Li Q and Wan JM (2005). SSRHunter: Development of a local searching software for SSR sites. Yi Chuan 27: 808-810. 
Lu HJ and Myers GO (2002). Genetic relationships and discrimination of ten influential upland cotton varieties using RAPD markers. Theor. Appl. Genet. 105: 325-331.

Pan L, Li Y, Guo R, Zhang FY, et al. (2014). Development of cpSSR markers in Vigna unguiculata and their transferability in related species. J. ChangJiang Vegetables 6: 9-15.

Pian RQ, Li W, Li N, Chen XY, et al. (2008). Development of microsatellite from complete sequence of Medicago truncatula chloroplast DNA. J. Anhui Agric. Sci. 36: 3531-3534.

Pillay M and Myers GO (1999). Genetic diversity in cotton assessed by variation in ribosomal RNA genes and AFLP markers. Crop Sci. 39: 1881-1886.

Powell W, Morgante M, McDevitt R, Vendramin GG, et al. (1995). Polymorphic simple-sequence repeat regions in chloroplast genomes: applications to the population genetics of pines. Proc. Natl. Acad. Sci. U. S. A. 92: 7759-7763.

Rana MK, Singh VP and Bhat KV (2005). Assessment of genetic diversity in upland cotton (Gossypium hirsutum L.) breeding lines by using amplified fragment length polymorphism (AFLP) markers and morphological characteristics. Genet. Resour. Crop Evol. 52: 989-997.

Raubeson LA, Peery R, Chumley TW, Dziubek C, et al. (2007). Comparative chloroplast genomics: analyses including new sequences from the angiosperms Nuphar advena and Ranunculus macranthus. BMC Genomics 8: 174.

Rose O and Falush D (1998). A threshold size for microsatellite expansion. Mol. Biol. Evol. 15: 613-615.

Smith JSC, Kresovich S, Hopkins MS, Mitchell SE, et al. (2000). Genetic diversity among elite sorghum inbred lines assessed with simple sequence repeats. Crop Sci. 40: 226-232.

Sugiura N, Kurokochi H, Tan E, Asakawa S, et al. (2014). Development of 13 polymorphic chloroplast DNA markers in Quercus gilva, a regionally endemic species in Japan. Conserv. Genet. Resour. 6: 961-965.

Tatineni V, Cantrell RG and Davis DD (1996). Genetic diversity in elite cotton germplasm determined by morphological characteristics and RAPDs. Crop Sci. 36: 186-192.

Vigouroux Y, Jaqueth JS, Matsuoka Y, Smith OS, et al. (2002). Rate and pattern of mutation at microsatellite loci in maize. Mol. Biol. Evol. 19: 1251-1260.

Wang HK, Qiao YS, Lou XM, Xue HB, et al. (2006). Distribution of microsatellite from complete sequence of Arabidopsis thaliana chloroplast DNA. Chin. J. Biochem. Mol. Biol. 22: 845-850.

Wang KB (2007). Introduction and conservation of wild cotton in China. Cotton Sci. 19: 354-361.

Wang KB, Wang ZW, Li FG, Ye WW, et al. (2012). The draft genome of a diploid cotton Gossypium raimondii. Nat. Genet. 44: 1098-1104.

Wang YL (2013). Chloroplast microsatellite diversity of Opisthopappus Shih (Asteraceae) endemic to China. Plant Syst. Evol. 299: 1849-1858.

Wendel JF and Albert VA (1992). Phylogenetics of the cotton genus (Gossypium): character-state weighted parsimony analysis of chloroplast-DNA restriction site data and its systematic and biogeographic implications. Syst. Bot. 17: 115-143.

Wendel JF and Brubaker CL (1993). RFLP diversity in Gossypium hirsutum L. and new insights into the domestication of cotton. Am. J. Bot. 80: 71.

Wendel JF, Rowley R and Stewart J McD (1994). Genetic diversity in and phylogenetic relationships of the Brazilian endemic cotton, Gossypium mustelinum (Malvaceae). Plant Syst. Evol. 192: 49-59.

Wu YX, Daud MK, Chen L and Zhu SJ (2007). Phylogenetic diversity and relationship among Gossypium germplasm using SSRs markers. Plant Syst. Evol. 268: 199-208.

$\mathrm{Xu}$ DH, Abe J, Gai JY and Shimamoto Y (2002). Diversity of chloroplast DNA SSRs in wild and cultivated soybeans: evidence for multiple origins of cultivated soybean. Theor. Appl. Genet. 105: 645-653.

Xue J, Wang S and Zhou SL (2012). Polymorphic chloroplast microsatellite loci in Nelumbo (Nelumbonaceae). Am. J. Bot. 99: 240-244.

Yang J, Li S, Sun G, Yuan Y, et al. (2008). Population structure and genetic variation in the genus Dipteronia Oliv. (Aceraceac) endemic to China as revealed by cpSSR analysis. Plant Syst. Evol. 272: 97-106.

Yu JZ, Fang DD, Kohel RJ, Ulloa M, et al. (2012). Development of a core set of SSR markers for the characterization of Gossypium germplasm. Euphytica 187: 203-213.

Zhang JF and Stewart JM (2000). Economical and rapid method for extracting cotton genomic DNA. J. Cotton Sci. 4: 193-201. 